\title{
Use of Premise-Reasoning-Outcome (PRO) in Structuring Students’ Answers for Open-Ended Questions
}

\author{
Ling Wee Lee, Goh Yinglun Allan \\ National Junior College (NJC), Singapore
}

\begin{abstract}
This paper describes the efficacy of premise-reasoning-outcome (PRO)—an instructional strategy to help students in scientific explanations. Students generally find it hard to answer open-ended "explain” type questions and it difficult to give a complete answer because of missing components or links in their answers. We used PRO as a structure for Grades 9 and 10 students in National Junior College (NJC) in Singapore, to answer open-ended questions. PRO stands for "premise" (the known facts, such as scientific laws, principles, and information related to the given scenario), "reasoning” (sequential chain of reasoning that links the "premise” to the "outcome”), and "outcome" (phenomenon to be explained). The effectiveness of the PRO approach was evaluated by using a survey of students' perception on the usefulness of PRO and a performance quiz on the efficacy of the PRO approach as a tool for students to provide complete answers to open-ended questions. In the implementation, $70 \%$ of the students have used the PRO structure in their explanations during a revision quiz at the end of the study with $94 \%$ of the students being able to identify the right premise(s) for the question. Students also responded positively to the use of PRO in helping them to structure their answers and they felt that the PRO structure is helpful for them.
\end{abstract}

Keywords: teaching science, science instruction, constructing scientific explanation, instructional strategies

\section{Introduction}

A main goal of science education is for students to develop a scientific understanding of the world through the use of the scientific method and reasoning, and not just a mastery of scientific concepts (Bricker \& Bell, 2009). The ability to engage in scientific argumentation, the explanation is as a core epistemic practice of science, and students have to learn to interpret context, analyze data, and question assumptions to come up with scientifically accepted explanations for any physical phenomenon (National Research Council [NRC], 1996, p. 113).

We have found that National Junior College (NJC) students generally find it hard to answer open-ended "explain" type questions with missing components or links in their explanations. We chanced upon the use of premise-reasoning-outcome (PRO) as an answering structure (Tang, 2015) and decided to utilize PRO as a structure to guide students in answering open-ended "explain" type questions. Using the PRO approach to explain a phenomenon, students will first state the principle or premise that is based on the scientific theory, follow up with a reasoning to explain why the phenomenon is happening based on the premise, and then give its outcome. We conducted a both a survey and a quiz to help us evaluate whether the students found the PRO structure was helpful in answering open-ended questions.

Ling Wee Lee, B.Sc (Hons), teacher, Physics Department,, National Junior College (NJC).

Goh Yinglun Allan, B.Sc (Hons), head of department, Physics Department, National Junior College (NJC). 


\section{Methodology}

The physics team started the process by teaching the Junior High 3 (JH 3 or Grade 9 equivalent) and Junior High 4 (JH 4 or Grade 10 equivalent) students, the PRO structure to answering open-ended questions that involves explanation. Specific topics were chosen for students to practice by using the PRO structure to answer questions in their tutorials. For JH 3, the topic is "Moments and stability (Please refer to Annex A)," while for JH 4, the topic is "Pressure (Please refer to Annex B)."

At the end of the academic year, the physics team came up with a quiz comprising three open-ended questions that require explanations, selected from different topics taught at the JH 3 level as part of students' revision. The three questions were chosen such that they come from different topics: "Newton's laws of motion, moments and stability, and work energy power."

Students answered the three questions on a Google Form (Please refer to Annex C) to aid in the collation of their answers. The students' answers were sorted and analyzed to find out whether the use of PRO has helped students give a more complete answer. The students also did a survey (Please refer to Annex D) on the effectiveness of the use of PRO to structure their answers.

\section{Results}

\section{Analysis on Students' Answers to Revision Quiz}

One hundred and eighty-four students from JH 3 and JH 4 levels completed a short revision quiz comprising of three questions involving concepts taught in the module PH 301 "The moving world," which covers mainly the mechanics portion of physics.

Teachers decided to zoom in on Question 3 (see Table 1) to analyze students' responses, because it is a relatively "standard" examination question commonly asked in the "O" level examination and this question involves a concept that was recently taught to the JH 3 students. Hence, the JH 3 students would be familiar to the concept tested in this question.

Table 1

PRO Marking Scheme for Grade 9 Question on Work Energy Power

\begin{tabular}{|l|l|}
\hline $\begin{array}{l}\text { Question 3: A woman holds on to a bowling ball tied to the ceiling. She releases the ball just below her chin. Would she be hit by } \\
\text { the ball when it swings back towards her? Explain your answer. }\end{array}$ \\
\hline Marking scheme & Elaboration of each reasoning point \\
\hline Mark for each reasoning point & $\begin{array}{l}\text { 1. By conservation of energy, final gravitational potential energy (GPE) must be } \\
\text { equal to the initial GPE. } \\
\text { 2. Due to friction or air resistance, some energy is lost as thermal energy as the ball } \\
\text { swings. Hence, the total mechanical energy of the ball decreases. }\end{array}$ \\
\hline Total energy of system & $\begin{array}{l}\text { At the highest point, total energy of the ball = GPE of the ball. The ball has only } \\
\text { GPE, since the velocity of ball = zero momentarily. }\end{array}$ \\
\hline $\begin{array}{l}\text { Comparison of final height and initial } \\
\text { height }\end{array}$ & Hence, the final height of the ball is equal to the initial height of the ball. \\
\hline
\end{tabular}



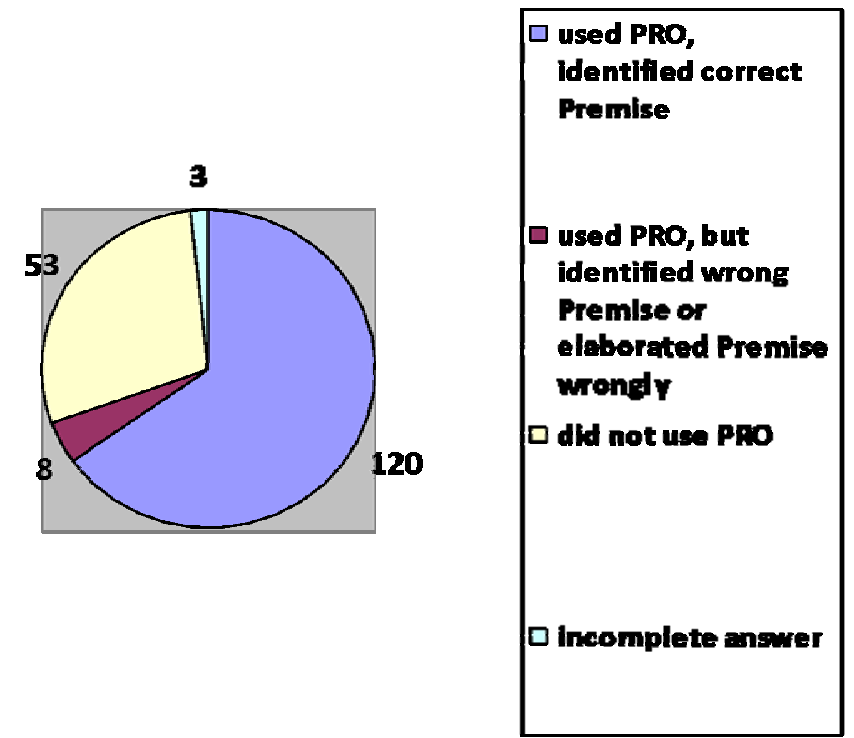

Figure 1. Students' response to question on work energy power.

Students' responses were collated and analyzed. As seen in Figure 1, 70\% of the students who attempted the online revision quiz used the PRO format in answering the questions. This $70 \%$ includes students who explicitly highlighted the "premise," "reasoning," and "outcome.” Also, those who clearly followed the structure by beginning their answers with "premise," followed by "reasoning," and then "outcome," without highlighting the components. About $6 \%$ of the students who used the PRO structure either chose the wrong premise, or elaborated wrongly on the premise (e.g., elaborated on energy conversion, instead of giving the statement of the principle of conservation of energy). These students generally did not do well as they had matched the question to the wrong concept, hence, their discussion would deviate from what is required.

There is no significant difference in terms of the quality of reasoning for students who used PRO and those who did not. Twenty-eight percent of students who used PRO and produced the right premise scored full marks based on the marks scheme, compared to 30\% of students who did not use PRO who also scored full marks.

An example of student's answer that scored full marks with PRO is as follows:

Premise: According to conservation of energy: Energy cannot be created or destroyed. It can only be converted from one form to another.

Reasoning: When the woman releases the ball, its GPE converts to kinetic energy (KE) and thermal energy (due to friction of the air), and when the KE converts to GPE when the ball swings back, part of the energy of the ball has been lost as thermal energy. GPE $=m g h, m$ and $g$ remain unchanged, $h$ decreases as GPE decreases.

Outcome: As a result, the ball can only reach a lower maximum height and will not hit the woman.

Without following the PRO structure, some students also weaved in the premise with their reasoning in their answers, such as in the example below. This example also scored full marks:

No, she would not. Because the GPE of the ball at the initial position is converted to KE as the ball moves forward, to GPE when it comes to instantaneous rest on the left, and to KE again as it moves back towards her. As energy will be lost as heat, by the law of conservation of energy, and given that GPE $=m g h$, with less energy converted back to GPE, the ball will not swing back as high and thus will not hit her.

For students who used the PRO structure to answer questions, 94\% are able to use the right premise for this question. Upon analysis of these students who used PRO in their answers, we found the number of marks 
awarded to the students is dependent on their ability to link the reasoning logically and sequentially from the premise to the outcome. Some students skipped links in their reasoning, while others failed to mention important keywords or revealed reasoning errors in their answers.

\section{Analysis on Students' Survey}

One hundred and fifty-three students completed the survey on the effectiveness of the use of PRO in answering questions. Students scored the survey questions on a 4-point Likert scale, from 1 ("Strongly disagree") to 4 (“Strongly agree”).

Survey questions:

1. I find PRO useful in helping me phrase written answers;

2. PRO helps me organize my thoughts and spot the gaps in my reasoning steps;

3. With PRO, I am confident that my answer is complete;

4. I understand the need to identify the right premise;

5. PRO helps me figure out the reasoning(s) and premise(s) that lead to the outcome;

6. PRO helps me make sense of new scenarios I encounter in physics;

7. I will consider using PRO as a thinking structure to better understand and explain phenomenon outside the physics classroom.

Most students consistently answered "Agree" and "Strongly agree" (70\% to $90 \%$ of the survey respondents) for all the questions.

Responses were sorted by levels and the average scores for the different questions reported by the JH 3 and JH 4 students were very similar. This suggests that the questions were well-phrased and the interpretation is unambiguous to the students surveyed both of the JH 3 and JH 4 levels.

Table 2

Likert Scale Rating (1 = "Strongly disagree” and 4 = "Strongly agree”) for Students' Perception of PRO

\begin{tabular}{llllllll}
\hline & Question 1 & Question 2 & Question 3 & Question 4 & Question 5 & Question 6 & Question 7 \\
\hline All & 3.02 & 3.05 & 2.84 & 3.27 & 3.03 & 2.80 & 2.89 \\
JH 3 & 3.02 & 3.07 & 2.88 & 3.25 & 3.04 & 2.82 & 2.92 \\
JH 4 & 3.03 & 3.04 & 2.83 & 3.29 & 3.03 & 2.80 & 2.88 \\
\hline
\end{tabular}

Questions 3, 6, and 7 have an average that falls below a rating of "3" (see Table 2). Further discussion with the students suggests that students are still not confident that their explanations are complete even with the use of PRO, and hence, they are unable to use PRO to make sense of new scenarios in physics, or to apply PRO outside of the physics classroom. Students' lack confidence and motivation in using and applying PRO might be due to a possible lack of practice in using the template. We observed that some students were still not able to correctly differentiate between the premise and reasoning during class discussion. Even with the understanding of PRO, some students faced difficulties in identifying related premises in explaining the context, or have trouble identifying the relevant concept(s) for the given scenario. Students also struggle when faced with complex problems involving more than one premise or links in reasoning.

\section{Key Results for Revision Quiz}

Out of the $70 \%$ of the students who used the PRO structure to answer questions and $94 \%$ are able to use identify the right premise for the question. 
However, there was no significant difference in terms of the quality of reasoning for students who used PRO and those who did not.

\section{Key Results for Students Survey}

1. Students responded positively to the use of PRO in helping them to structure their answers. They feel that the PRO structure is helpful for them;

2. Students however need to develop greater confidence in applying the PRO to answer questions.

\section{Conclusion and Reflection}

Though the results of the revision quiz showed that students are able to get full marks even without following the PRO structure, the teachers feel that there is value in teaching students a systematic way in answering questions. While some students did not use the PRO explicitly in answering questions, there might be benefits in familiarizing students with the PRO structure to help remind them to include the premise in their explanation. Depending on the mark scheme, some questions might award students a mark for simply stating the correct premise. However, we are unable to know if students are more likely to include a premise after being taught PRO, because we did not do a pre-assessment or post-assessment for comparison.

One of the challenges of teaching and learning the PRO structure is the uncertainty in determining what counts as a gapless explanation, especially for the students. As mentioned in the analysis, students did not score full marks for reasoning mainly, because there were missing links, which means missing reasoning points when compared to the mark scheme. However, not all science/physics teachers might even agree on the mark scheme — some might require less reasoning or marking points while others might demand more. A pragmatic approach to this is often based on number of marks awarded for the question, but this is only applicable in exam conditions as we rarely give breakdown of marks for tutorial or practice questions.

Future work for investigation might include matching students' response to their quiz score to see whether having the PRO structure might benefit any specific groups of students (e.g., the higher or lower ability students).

\section{References}

Achinstein, P. (1983). The nature of explanation. New York, N.Y.: Oxford University Press.

Braaten, M., \& Windschitl, M. (2011). Working toward a stronger conceptualization of scientific explanation for science education. Science Education, 95(4), 639-669.

Bricker, L., \& Bell, P. (2009). Conceptualizations of argumentation from science studies and the learning sciences and their implications for the practices of science education. Science Education, 92(3), 473-498.

Bybee, R. W., Taylor, J. A., Gardner, A., Van Scotter, P., Powell, J. C., \& Westbrook, A. (2006). The BSCS 5E instructional model: Origins and effectiveness. Colorado Springs, C.O.: BSCS.

Dagher, Z., \& Cossman, G. (1992). Verbal explanations given by science teachers: Their nature and implications. Journal of Research in Science Teaching, 29(4), 361-374.

Halliday, M. A. K., \& Martin, J. R. (1993). Writing science: Literacy and discursive power. Pittsburgh, P.A.: University of Pittsburgh Press.

National Research Council (NRC). (1996). National science education standards. Washington, D.C.: National Academies Press.

Salmon, W. C. (1989). Four decades of scientific explanation. Minneapolis, M.N.: University of Minnesota Press.

Sandoval, W. A. (2003). Conceptual and epistemic aspects of students' scientific explanations. Journal of the Learning Sciences, 12(1), 5-51.

Tang, K. S. (2015). The PRO instructional strategy in the construction of scientific explanations. Teaching Science, 61(4), 14-21. Wellington, J., \& Osborne, J. (2001). Language and literacy in science education. Philadelphia, P.A.: Open University Press. 